\title{
Studies on the Mean Zonal Flow I
}

\author{
By Y. Ishimaru
}

(Manuscript received 13 Sept., 1957)

\begin{abstract}
In this paper, the author treated the distribution of the Zonal wind with constant absolute vorticity, and gave some remarks on the position of the jet-stream. The vertical relative vorticity and its distribution on the globe is discussed in detail. The author's relations contain Rossby's and Kasahara's results as its special cases.
\end{abstract}

\section{Introduction}

In 1947 , C.-G. Rossby ${ }^{1)}$ showed that the profile of the mean zonal wind observed at the tropopause level agrees fairly well with the theoretical profile computed on the assumption that the lateral large-scale turbulence leads to the equalization of the vertical component of absolute vorticity north of the jet-stream, i.e. in the polar cap. According to Rossby's theory, the sharp breakdown of the zonal wind profile south of the jet stream is the consequence of mechanically generated inertia instability which leads to the development of the meridional circulation. And, the profile of the mean zonal wind on the southern side of the jet-stream is derived on the assumption of the constant absolute angular momentum. Later A. Kasahara ${ }^{2)}$ treated the same problem on the assumption that the uniform distribution of the vertical component of the absolute vorticity on each side of the jet-stream. The above two assumptions correspond to the meridional circulation theory and the lateral mixing theory in the tropical region respectively.

In the present paper, the author treated the same problem more generally on the assumption of uniform distribution of the vertical component of the absolute vorticity. Various special cases are discussed in detail, and our result includes Rossby's and Kasahara's results as its special cases.

\section{The distribution of the zonal wind with a constant absolute vorticity}

If the vertical component of the absolute vorticity $\eta$ is a function of $\varphi$ only, it is ex- pressed by

$$
\eta=-\frac{1}{a} \frac{\partial V_{E}}{\partial \varphi}+\frac{V_{E}}{a} \operatorname{tg} \varphi+2 \varepsilon \sin \varphi
$$

where $V_{t}, a$ and $\varepsilon$ are the zonal velocity, the radius and the angular velocity of the earth respectively. Then the solution of zonal motion $V_{E}$ may be written in the following form:

$$
V_{E}=\frac{1}{\cos \varphi}\left\{\int(2 \varepsilon a \sin \varphi-\eta a) \cos \varphi d \varphi+c\right\},
$$

where $c$ is an integration constant.

Therefore, if $\eta$ is constant, $V_{E}$ is given by

$$
V_{E}=-\varepsilon a \cos \varphi-\eta a \operatorname{tg} \varphi+\frac{c}{\cos \varphi} .
$$

The zonal velocity distribution is assumed to have the absolute vorticity $\eta_{p}$ in the polar cap and $\eta_{a}$ in the tropical region. If $V_{E}$ is finite at the pole $(\varphi=\pi / 2)$, the integration constant is easily determined as $C=\eta_{p} a$; and if $V_{E}$ is $C_{B}$ at the equator $(\varphi=0), C=\varepsilon a+C_{E}$; then we have

$$
\begin{gathered}
V_{E}=-\varepsilon a \cos \varphi+\frac{\eta_{p} a}{\cos \varphi}(1-\sin \varphi) \\
\text { in the polar cap, } \\
V_{B}=-\varepsilon a \cos \varphi-\eta_{a} a \operatorname{tg} \varphi+\frac{\varepsilon a+C_{H}}{\cos \varphi} \\
\text { in the tropical region. }
\end{gathered}
$$

At the latitude $\varphi_{0}$ of the axis of the jetstream, the velocity should be the same. Then we have

$$
\begin{aligned}
V_{E_{0}} & =-\varepsilon a \cos \varphi_{0}+\frac{\eta_{p} a}{\cos \varphi_{0}}\left(1-\sin \varphi_{0}\right) \\
& =-\varepsilon a \cos \varphi_{0}-\eta_{a} a \operatorname{tg} \varphi_{0}+\frac{\varepsilon a+C_{t}}{\cos \varphi_{0}}
\end{aligned}
$$




$$
\left.\begin{array}{l}
\eta_{p}=\left(\varepsilon+\frac{V_{E_{0}}}{a \cos \varphi_{0}}\right)\left(1+\sin \varphi_{0}\right), \\
\eta_{a}=\varepsilon \sin \varphi_{0}+\frac{C_{E}-V_{E_{0}} \cos \varphi_{0}}{a \cos \varphi_{0}}
\end{array}\right\}
$$

Here, $V_{E_{0}}$ is the velocity on the axis of the jet-stream and $C_{E}$ has the negative sign in order to be prevailing equatorial easterly wind. Therefore,

$$
\begin{aligned}
& V_{E}=-\varepsilon a \cos \varphi \\
&+\frac{(1-\sin \varphi) \cos \varphi_{0}}{\left(1-\sin \varphi_{0}\right) \cos \varphi}\left(V_{E_{0}}+\varepsilon a \cos \varphi_{0}\right) \\
& \text { in the polar cap, } \\
& V_{E}=\frac{\varepsilon a \sin ^{2} \varphi+C_{E}}{\cos \varphi} \\
&-\left(\frac{\varepsilon a \sin ^{2} \varphi_{0}+C_{F}}{\cos \varphi_{0}}-V_{E_{0}}\right) \frac{\operatorname{tg} \varphi}{\operatorname{tg} \varphi_{0}} \\
& \text { in the tropical region. }
\end{aligned}
$$

It is not probable that the polar cap would have a larger absolute vorticity than $2 \varepsilon$. Thus, if we put

$$
\eta_{p}=n \varepsilon,
$$

Then (8) may be written as follows:

$$
\begin{aligned}
& V_{E}=\varepsilon a \cos \varphi(\left.\frac{n}{1+\sin \varphi}-1\right), \\
& \text { in the polar cap, } \\
& V_{E}= \varepsilon a \cdot \operatorname{tg} \varphi\left(\sin \varphi+\frac{n\left(1-\sin \varphi_{0}\right)-1}{\sin \varphi_{0}}\right) \\
&+ \frac{C_{E}}{\cos \varphi}\left(1-\frac{\sin \varphi}{\sin \varphi_{0}}\right), \\
& \text { in the tropical region. }
\end{aligned}
$$

If $n$ is equal to or larger than $2, V_{E}$ is always positive. On the other hand, $n$ is smaller than 2, there is a zone of negative $V_{E}$ i.e. polar easterlies.

Now, if we assume that in the region between the latitude $\varphi_{1}$ and the pole the wind is easterly and between the latitudes $\varphi_{0}$ and $\varphi_{1}$ the wind is westerly, and if $V_{E}=0$ at $\varphi=\varphi_{1}$, then from Eq. (10) (i), we have

$$
n=1+\sin \varphi_{1}
$$

Generally,

$$
\begin{gathered}
V_{E}=\varepsilon a \cos \varphi\left(\frac{\sin \varphi_{1}-\sin \varphi}{1+\sin \varphi}\right), \quad \frac{\pi}{2} \geq \varphi \geq \varphi_{0} \\
\text { in the polar cap, }
\end{gathered}
$$

$$
V_{E}=\varepsilon a \cos \varphi_{0}\left(\frac{\sin \varphi_{1}-\sin \varphi_{0}}{1+\sin \varphi_{0}}\right), \quad \varphi=\varphi_{0}
$$

on the axis of the jet-stream,

$$
V_{E}=\varepsilon_{a} \operatorname{tg} \varphi\left(\sin \varphi-\sin \varphi_{1}+\frac{\sin \varphi_{1}}{\sin \varphi_{0}}-1\right)
$$

$$
+\frac{C_{E}}{\cos \varphi}\left(1-\frac{\sin \varphi}{\sin \varphi_{0}}\right)
$$$$
\varphi_{0} \geq \varphi \geq 0 \text { in tropical latitudes, (iii) }
$$

in which $\varphi_{1}>\varphi_{0}$.

The constant absolute vorticities are given by

$$
\begin{aligned}
& \eta_{p}=\varepsilon\left(1+\sin \varphi_{1}\right)=n \varepsilon, \\
& \eta_{a}=\varepsilon\left(\left(1+\sin \varphi_{1}\right)-\frac{\sin \varphi_{1}}{\sin \varphi_{0}}\right)+\frac{C_{E}}{a \sin \varphi_{0}} .
\end{aligned}
$$

Next, from (12) (iii) we find the latitude $\varphi_{0}$ where the velocity becomes zero in the tropical region, that is

$$
\begin{gathered}
\varepsilon a \sin \varphi_{2}\left(\sin \varphi_{2}-\sin \varphi_{1}+\frac{\sin \varphi_{1}}{\sin \varphi_{0}}-1\right) \\
+C_{E}\left(1-\frac{\sin \varphi_{2}}{\sin \varphi_{0}}\right)=0
\end{gathered}
$$

From the above equation, we get

$$
\begin{aligned}
C_{E}= & -\varepsilon a \sin \varphi_{2}\left\{\left(1-\sin \varphi_{0}\right)\right. \\
& \left.\times \frac{\sin \varphi_{1}-\sin \varphi_{0}}{\sin \varphi_{0}-\sin \varphi_{2}}-\sin \varphi_{0}\right\},
\end{aligned}
$$

or

$$
\sin \varphi_{0}=\frac{\sin \varphi_{1}-\frac{C_{E}}{\varepsilon a}}{1+\sin \varphi_{1}-\sin \varphi_{2}-\frac{C_{B}}{\varepsilon a \sin \varphi_{2}}},
$$

and

$$
\eta_{a}=\varepsilon\left(\sin \varphi_{2}+\frac{C_{E}}{\varepsilon a \sin \varphi_{2}}\right) .
$$

We can see that the constant absolute vorticities in the polar cap or in the tropical region are determined by the positions where the zonal velocity vanishes in each region respectively. 
3. The special distributions of zonal wind

(i) When the absolute vorticity in the tropical region is zero, i.e. the absolute angular momentum is constant, and that in the polar cap is constant, viz.

$$
\eta_{a}=0 \quad \text { and } \quad \eta_{p}=\varepsilon\left(1+\sin \varphi_{1}\right),
$$

we have, from (16),

$$
C_{E}=-\varepsilon a \sin ^{2} \varphi_{2},
$$

which shows that the equatorial easterly wind blows. Substituting the relation (17) in (15), we get

$$
\sin ^{2} \varphi_{2}=\left(1+\sin \varphi_{1}\right) \sin \varphi_{0}-\sin \varphi_{1} .
$$

Then, the zonal wind distribution is as follows:

$$
\begin{aligned}
& V_{E}=\varepsilon a \cos \varphi\left(\frac{\sin \varphi_{1}-\sin \varphi}{1+\sin \varphi}\right), \\
& \text { in the polar cap, } \\
& V_{E}=\varepsilon a\left(\frac{\cos ^{2} \varphi_{2}}{\cos \varphi}-\cos \varphi\right) \\
& =\varepsilon a\left\{\frac{1-\left(1+\sin \varphi_{1}\right) \sin \varphi_{0}+\sin \varphi_{1}}{\cos \varphi}-\cos \varphi\right\} \text {, } \\
& \text { in the tropical region. }
\end{aligned}
$$

When the westerly winds prevail in all over the polar cap, $\varphi=\pi / 2$, then $\eta_{p}=2 \varepsilon$. Therefore, (19) may be written by

$$
\begin{gathered}
V_{E}=\varepsilon a \cos \varphi\left(\frac{1-\sin \varphi}{1+\sin \varphi}\right), \quad \frac{\pi}{2} \geq \varphi \geq \varphi_{0} \\
\text { in the polar cap, } \\
V_{E}=\varepsilon a\left\{\frac{2\left(1-\sin \varphi_{0}\right)}{\cos \varphi}-\cos \varphi\right\}, \varphi_{0} \geq \varphi \geq 0 \\
\text { in the tropical region, }
\end{gathered}
$$

in which

$$
\sin ^{2} \varphi_{2}=2 \sin \varphi_{0}-1
$$

and

$$
C_{E}=-\varepsilon a\left(2 \sin \varphi_{0}-1\right) .
$$

This solution was pointed out by Rossby (1947).

Then, if $\varphi_{2}=0$, in the tropical region no easterly wind zone exists and the equatorial current is zero. In this case, we find the position of jet-stream as follows: $\sin \varphi_{0}=1 / 2$ or $\varphi_{0}=30^{\circ}$.
In we can use the value $\varphi_{2}$ from the radiational heat balance (See Appendix II in part II) or a distribution of the surface temperature due to the conduction of heat as used by Oberbeck and others, we have

$$
1-3 \sin ^{2} \varphi_{2}=0 \quad \text { or } \quad \varphi_{2}=35^{\circ} 16^{\prime} \text {. }
$$

Then, we get

$$
\sin \varphi_{0}=\frac{2}{3} \quad \text { or } \quad \varphi_{0}=41^{\circ} 49^{\prime}
$$

and from $\varepsilon a=463 \mathrm{~m} / \mathrm{sec}$, the velocity of the equatorial easterly wind is given as $\varepsilon a / 3=154$ $\mathrm{m} / \mathrm{sec}$, and maximum westerly in the jetstream is $\varepsilon a / 3 \sqrt{5}=69 \mathrm{~m} / \mathrm{sec}$.

From the above equation, some examples of zonal wind distribution in the both regions are given in Fig. 1.

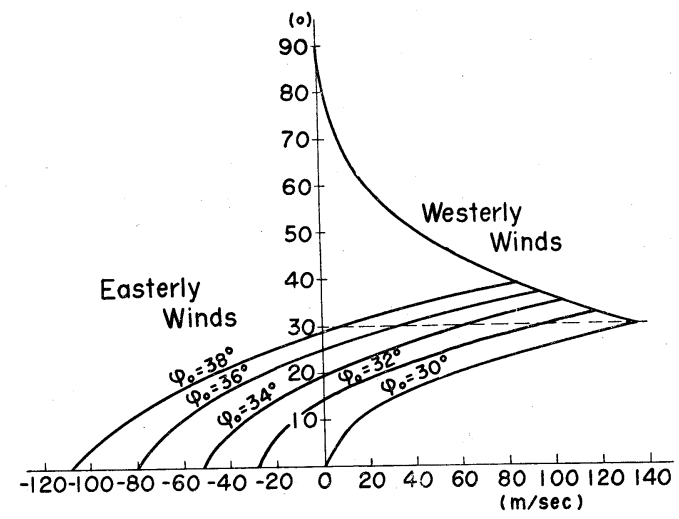

Fig. 1. Some zonal wind profiles in tropopause level.

(ii) When the absolute vorticities are constant in the polar cap and in the tropical region, and the zonal wind velocity at the equator is zero by the assumption of symmetrical distribution of pressure in both hemispheres, that is

$$
\eta_{a}=\varepsilon \sin \varphi_{2}, \quad \eta_{p}=\varepsilon\left(1+\sin \varphi_{1}\right)
$$

and

(15) is

$$
C_{E}=0
$$
(15) is

$$
\left.\begin{array}{l}
\sin \varphi_{0}=\frac{\sin \varphi_{1}}{1+\sin \varphi_{1}-\sin \varphi_{2}} \\
\sin \varphi_{2}=\left(1+\sin \varphi_{1}\right)-\frac{\sin \varphi_{1}}{\sin \varphi_{0}} .
\end{array}\right\}
$$

Then, the zonal wind velocity is given by 


$$
\begin{aligned}
& V_{E}=\varepsilon a \cos \varphi\left(\frac{\sin \varphi_{1}-\sin \varphi}{1+\sin \varphi}\right), \\
& \quad \text { in the polar cap, } \\
& V_{E}=\varepsilon a \operatorname{tg} \varphi\left(\sin \varphi-\sin \varphi_{2}\right) \\
& =\varepsilon a \operatorname{tg} \varphi\left(\sin \varphi-\sin \varphi_{1}+\frac{\sin \varphi_{1}}{\sin \varphi_{0}}-1\right),
\end{aligned}
$$$$
\text { in the tropical region. (ii) }
$$

From the above equations, the zonal wind distribution in both regions in case $\varphi_{0}=38^{\circ}$ is illustrated in Fig. 2.

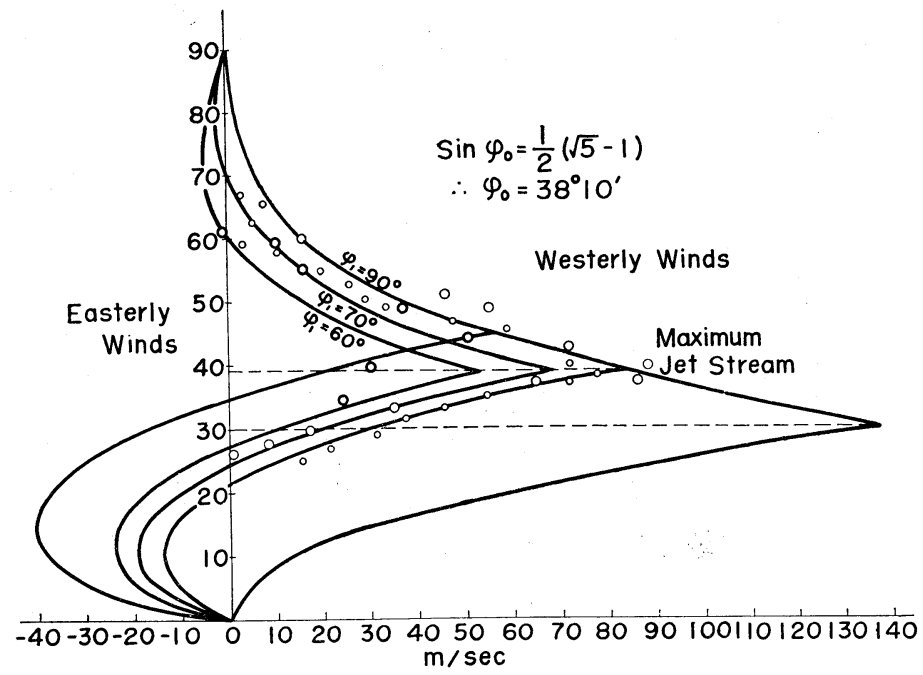

Fig. 2. Comparison between the theoretical wind profile of jetstream and a few observed zonal wind profiles from the tropopause level over North America (modified after Willet and Rossby.

a) When the westerly winds prevail in the whole hemisphere, we have

$$
\eta_{a}=0 \quad \text { and } \quad \eta_{p}=2 \varepsilon .
$$

Therefore,

$$
\begin{array}{ll}
V_{E}=\varepsilon a \cos \varphi \frac{1-\sin \varphi}{1+\sin \varphi}, & \frac{\pi}{2} \geq \varphi \geq \varphi_{0} \\
V_{E}=\varepsilon a \operatorname{tg} \varphi \sin \varphi, & \varphi_{0} \geq \varphi \geq 0
\end{array}
$$

in which

$$
\sin \varphi_{0}=\frac{1}{2}, \quad \varphi_{0}=30^{\circ}
$$

and

$$
\varphi_{1}=90^{\circ}, \quad \varphi_{2}=0^{\circ} .
$$

b) When the westerly winds prevail in the polar cap, we have

$$
\eta_{a}=\varepsilon \sin \varphi_{2} \quad \text { and } \quad \eta_{p}=2 \varepsilon .
$$

Then, the westerly wind distribution in the polar cap is the same as shown in (24) (i), but the zonal wind distribution in the tropical region is shown as follows

$$
\begin{aligned}
V_{E} & =\varepsilon a\left(\sin \varphi-\sin \varphi_{2}\right) \operatorname{tg} \varphi \\
& =\varepsilon a\left(\sin \varphi+\frac{1}{\sin \varphi_{0}}-2\right) \operatorname{tg} \varphi,
\end{aligned}
$$

in which $\sin \varphi_{0}=1 /\left(2-\sin \varphi_{2}\right)$ and $\varphi_{1}=90^{\circ}$.

This solution is the same as that obtained by Kasahara.

\section{Some remarks on the po- sition of jet-stream}

i) Profile of Vorticity

In the case $C_{E}=0$, the value of absolute vertical vorticity $\eta_{a}$ in the tropical region is as follows:

$$
\begin{aligned}
\frac{\eta_{a}}{\eta_{p}} & =\frac{\sin \varphi_{2}}{1+\sin \varphi_{1}} \\
& =1-\frac{\sin \varphi_{1}}{\left(1+\sin \varphi_{1}\right) \sin \varphi_{0}} .
\end{aligned}
$$

This is a functions of latitude $\varphi_{1}$ and $\varphi_{2}, \varphi_{0}$ and $\varphi_{1}$ or $\varphi_{0}$ and $\varphi_{2}$. If we know two positions of the latitudes $\varphi_{0}, \varphi_{1}$ or $\varphi_{2}$, we can determine $\eta_{a} / \eta_{p}$.

On the other hand, if we know $\eta_{a} / \eta_{p}$ or the position of jetstream, we can get $\varphi_{1}$ and $\varphi_{2}$.

ii) Position of Jet-stream

If we know $\varphi_{1}$ and $\varphi_{2}$, we can easily obtain the position of jet-stream by (18) or (21).

If the polar cap has no easterly wind, $\varphi_{1}$ is $90^{\circ}$, and we have

$$
\sin \varphi_{0}=\frac{1}{2-\sin \varphi_{2}}
$$

which is given in the following table.

Table 1. The relation between $\varphi_{2}$ and the latitude of the axis of the jet-stream.

\begin{tabular}{c|c|c|c|c|c|c|c|c|c}
\hline$\varphi_{2}$ & $0^{\circ}$ & $5^{\circ}$ & $10^{\circ}$ & $15^{\circ}$ & $20^{\circ}$ & $25^{\circ}$ & $30^{\circ}$ & $35^{\circ}$ \\
\hline$\varphi_{0}$ & $30^{\circ}$ & $31.5^{\circ}$ & $33.2^{\circ}$ & $35.0^{\circ}$ & $37.1^{\circ}$ & $39.4^{\circ}$ & $41.8^{\circ}$ & $44.5^{\circ}$ \\
\hline
\end{tabular}

Therefore, if the region of tropical easterly 
wind varies from $\varphi_{2}=0^{\circ}$ to $\varphi_{2}=35^{\circ}$, the position of jet-stream varies from $\varphi_{0}=30^{\circ}$ to $\varphi_{0}$ $=44.5^{\circ}$.

The continuous solution of zonal wind in the hemisphere is given when $\eta=\varepsilon$. If we put $V_{E}=0$ at the pole and the equator, from (3), we have the following distribution of zonal wind.

$$
V_{E}=\varepsilon a(\sin \varphi-1) \operatorname{tg} \varphi .
$$

This continuous solution of zonal wind represents easterlies.

Now, we can find the maximum velocity of this zonal wind. From the relation:

$$
\left|\frac{\partial V_{E}}{\partial \varphi}\right|_{\varphi=\varphi_{0}}=\varepsilon a\left|\frac{\partial}{\partial \varphi}[(\sin \varphi-1) \operatorname{tg} \varphi]\right|_{\varphi=\varphi_{0}}=0,
$$

in which $\varphi_{0}$ is the position of jet-stream, we get

$$
\left|\frac{\sin \varphi-1}{\cos ^{2} \varphi}+\sin \varphi\right|_{\varphi=\varphi_{0}}=0
$$

or

$$
\sin ^{2} \varphi_{0}+\sin \varphi_{0}-1=0
$$

then

$$
\left.\begin{array}{c}
\sin \varphi_{0}=\frac{1}{2}(\sqrt{5}-1)=0.6180 \\
\varphi_{0}=38^{\circ} 10^{\prime},
\end{array}\right\}
$$

which has already been obtained by $\mathrm{H}$. Arakawa $^{3)}$.

According to Willett ${ }^{4}$, a pronounced maximum of westerly winds (jet-stream) is at the height of tropopause somewhere between $30^{\circ} \mathrm{N}$ and $50^{\circ} \mathrm{N}$ over North America, in which the maximum is at about $31^{\circ} \mathrm{N}$ in winter and at $50^{\circ} \mathrm{N}$ in summer. The most probable position is at $38^{\circ} \mathrm{N}$ as shown in Fig. 2, and this fact shows a good agreement between the observation data and our calculation.

\section{The vertical relative vorticity}

If $\zeta$ denotes the vertical relative vorticity and is a function of latitude only, it must be written as follows:

$$
\zeta=-\frac{1}{a} \frac{\partial V_{E}}{\partial \varphi}+\frac{V_{E}}{a} \operatorname{tg} \varphi .
$$

From (12), $\zeta$ is given by

$$
\begin{array}{r}
\zeta_{p}=-\varepsilon\left(2 \sin \varphi-\sin \varphi_{1}-1\right), \\
\frac{\pi}{2} \geq \varphi \geq \varphi_{0} \\
\zeta_{a}=-\varepsilon\left(2 \sin \varphi-\sin \varphi_{2}-\frac{C_{E}}{\varepsilon a \sin \varphi_{2}}\right), \\
\varphi_{0} \geq \varphi \geq 0
\end{array}
$$

in which $\zeta_{p}$ is the vertical relative vorticity in the polar cap and $\zeta_{a}$ in the tropical region.

Then, in the polar region the negative relative vorticity region exists between the pole and $\sin \varphi_{3}=\left(1+\sin \varphi_{1}\right) / 2$ in which $\varphi_{3}$ is the latitude of the position of polar front and the positive relative vorticity must exist between $\varphi_{3}$ and the position of jet-stream $\left(\varphi_{0}\right)$.

When $C_{E}=0$, in the tropical region the negative vorticity exists between $\varphi_{0}$ and $\sin \varphi_{4}$ $=\sin \varphi_{2} / 2$ in which $\varphi_{4}$ is the latitude of the position of equatorial front and the positive relative vorticity must exist between $\varphi_{4}$ and the equator.

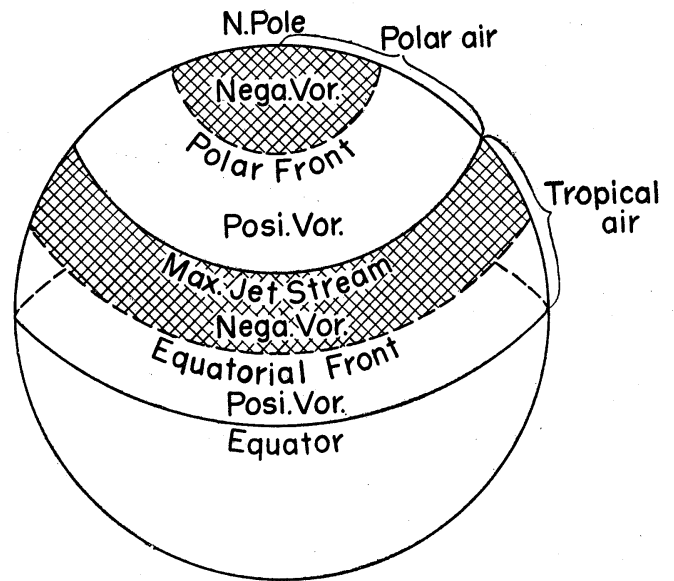

Fig. 3. Relative vorticity distribution on the Globe.

As the position of the jet-stream is at $\varphi_{0}$ $=38^{\circ} 10^{\prime}$ and the position where the tropical easterly winds change to the westerly is at $\varphi_{2}=35^{\circ} 16^{\prime}$, we find that $\varphi_{1}=43.1^{\circ}, \varphi_{3}=57.3^{\circ}$ and $\varphi_{4}=16.8^{\circ}$ as shown in Fig. 3. Next, for $\varphi_{0}=38^{\circ} 10^{\prime}$ and no easterly wind around the polar cap, the limiting position of $\varphi_{2}$ is $22.5^{\circ}$ and $\varphi_{4}$ is $11.0^{\circ}$. In this case the negative vorticity region exists around the polar cap in which the positive relative a vorticity is concentrated in latitudes between $38^{\circ} 10^{\prime}$ and $11^{\circ}$.

$\mathrm{S}$. Syōno ${ }^{5)}$ found a negative vorticity region in a typhoon, and formulated the theory that, 
if the positive relative vorticity is concentrated in finite region, the negative relative vorticity must be produced around it. It seems that this mechanism is applicable to the general circulation of the atmosphere. (continue)

\section{References}

1. C.-G. Rossby (1947): On the Distribution of Angular Vorticity in Gaseous Envelopes Under the Influence of Large Scale Horizontal Mixing Processes. Bull. Amer. Met. Soc., 28, No. 2, pp. 53-68.
2. Kasahara (1950): On the Dynamical Mechanism of the High Tropospheric Jet-Stream. Geophys. Notes, 3, No. 31, Geophys. Inst. Tokyo Univ.

3. H. Arakawa (1941): Über die Potentille Rotor (Potential Vorticity), Journ. Met. Soc. Japan, 19, No. 5.

4. H. C. Willett (1944): Descriptive Meteorology. New York, Academic Press.

5. S. Syōno (1951): "On the Dynamics of Cyclones", Geophys. Notes, 1, No. 7 (1950) and "On the Structure of Atmospheric Vortices", Journ. Met., 8, No. 2, p. 103. 